Pacific Journal of Mathematic 


\title{
QUASIREGULAR, PSEUDOCOMPLETE, AND BAIRE SPACES
}

\author{
AARON R. TODD
}

\begin{abstract}
J. C. Oxtoby obtains the essence of the classical Baire category theorems in his pseudocomplete spaces. In doing so, he reduces the role of points in his auxilary definitions of quasiregular spaces and pseudobases. With a natural modification of his definition of pseudobase that continues this reduction, a topological space is quasiregular if it has a pseudobase of closed sets. With this change, strong nesting is explicitly required in the definition of pseudocomplete spaces. The change also leads to an equivalence relation on the topologies of a set $X$ : Topologies $\sigma$ and $\tau$ are $S$ related if $\tau^{*}=\tau \backslash\{\phi\}$ is a pseudobase for $\sigma$. It also leads to conditions for which a topology finer or coarser than a pseudocomplete topology is itself pseudocomplete. Several examples illustrate the utility of quasiregularity, and there is a discussion of extensions of topological spaces. In particular, it is noted that a $T_{1}$-space is quasiregular iff it has a quasiregular compactification, and a topological space has a quasiregular one-point compactification iff the space has pseudobase of closed compact sets.
\end{abstract}

1. Introduction. In the concept of pseudocompleteness, Oxtoby [16] effectively distilled the major features of the proofs of the classical Baire category theorems. Often authors who find conditions implying that a space is a Baire space could as easily have shown that the conditions imply the stronger property of pseudocompleteness. In particular, the proofs of the Baire category theorems of Hajek [6] and McCoy [15] could as well serve to give the following results respectively: (1) A relatively Hausdorff compactification $\langle Y, f\rangle$ of a regular space $X$ is pseudocomplete, and (2) A quasiregular lightly compact space is pseudocomplete. Such results and necessary definitions are discussed later.

Since a pseudocomplete linear metrizable space is a completely metrizable space (Todd [19]), and an infinite dimensional Banach space has proper dense linear subspaces that are Baire (Kelley, Namioka, et al. [11] p. 95, prob. 10.B) pseudocompleteness strictly implies the Baire property. Another way of observing the difference: A product of pseudocomplete spaces is pseudocomplete (Oxtoby [16]) and there exist (nonempty) Baire spaces with squares that are not Baire. The existence of such Baire spaces has an interesting literature (see [16], [1] and especially [4] and [17] for citations).

Other permanence results for pseudocompleteness often resemble those for Baire, yet there are simple results for the Baire property 
that are only partially known for pseudocompleteness. Aarts and Lutzer [1] raise questions on two such results: (1) Is the continuous open image of a pseudocomplete space, pseudocomplete, and (2) Is a dense $G_{\tilde{o}}$-subspace of a pseudocomplete space, pseudocomplete? A partial result for the latter appeared in Oxtoby ([16], 5.2): If $Y$ is a quasiregular space with a pseudobase $\mathscr{B}$ consisting of sets whose closure is countably compact, then any dense $G_{\dot{o}}$-subspace $X$ of $Y$ is pseudocomplete. Neither question is fully answered here, however, Theorem 4.1 gives an improvement on Oxtoby's result and Corollary 3.8 is cast as a start on the first question.

The fastest definition of a Baire space is the following: A topological space is a Baire space if the intersection of each countable family of dense open sets is dense. If such intersections are nonempty the space is nonmeager. If the space is meager, then by complementation the space is the union of a countable family of rare sets (a set is rare if its closure has empty interior). Fleissner and Kunen [4] give a brief indication of the usefulness of Baire space throughout mathematics.

Quasiregularity and pseudobases, defined in Oxtoby [16], can be seen as usefully reducing the emphasis on points in topology and directing attention to collections of sets with close relationships to the family of open sets: A topological space $(X, \tau)$ is quasiregular if each nonempty open set $U \in \tau^{*}=\tau \backslash\{\phi\}$ contains the closure of a nonempty open set $V \in \tau^{*}$. That this may be a useful variation on regularity is suggested by the following: A regular $H$-closed space is necessarily compact (Willard [20], p. 127, prob. 17 K.3). However, a quasiregular $H$-closed space need not be compact as shown by Example 2.5. Nonetheless, it is pseudocompact as shown in $\S 2$.

Oxtoby [16] requires that a pseudobase be a collection $\mathscr{B}$ of nonempty open sets such that each nonempty open set contains a member of $\mathscr{B}$. We further deemphasize points by the following definition of pseudobase.

DEFINITION 1.1. A collection $\mathscr{B}$ of subsets of a topological space $(X, \tau)$ is a pseudobase of the topology $\tau$ if (i) for each $B$ in $\mathscr{B}$, int $\tau \neq \phi$ and (ii) for each $U$ in $\tau^{*}$, there is $B$ in $\mathscr{B}$ with $B$ contained in $U$.

This definition leads to an equivalence relation on the family of topologies on a given set $X$ : Topologies $\sigma$ and $\tau$ on $X$ are in this relation if and only if $\tau^{*}$ is a pseudobase for $\sigma$. It turns out that if one member of an equivalence class is Baire, then all members are Baire. The definition also simplifies descriptions of properties of spaces in terms of properties of members of a pseudobase. For 
example, the quasiregular property is immediately seen to be equivalent to the existence of a pseudobase of closed sets. Also see the result of Oxtoby quoted above and, of course, results given throughout this paper.

The definition of pseudobase entails a change in the definition of pseudocomplete spaces:

Definition 1.2. A topological space $(X, \tau)$ is pseudocomplete if it is quasiregular and there is a sequence $\left(\mathscr{B}_{n}\right)$ of pseudobases for $\tau$ such that $\bigcap_{n} B_{n} \neq \phi$, whenever $B_{n}$ is in $\mathscr{B}_{n}$ and int $B_{n}$ contains $\operatorname{cl}_{\tau} B_{n+1}$ for $n=1,2,3, \cdots$.

If a pseudobase is required to consist of open sets, as in Oxtoby's definition of pseudobase, then the strong nesting property int $_{\tau} B_{n} \supset \mathrm{cl}_{\tau} B_{n+1}$ in Definition 1.5 becomes, simply, $B_{n} \supset \mathrm{cl}_{\tau} B_{n+1}$ giving Oxtoby's original definition of pseudocompleteness. Therefore Oxtoby's original property is formally stronger than this notion of pseudocompleteness. Whether or not it is strictly stronger is an open question. The results of this paper suggest that the present notion of pseudocompleteness is an appropriate and interesting answer to the unification problem discussed in Aarts and Lutzer [2]. Is there a natural class of spaces that contains all completely metrizable spaces and all locally compact Hausdroff spaces and for which the conclusion of the Baire category theorem holds?

The second of the following is a formal result, the others follow from standard arguments found in Oxtoby [16] (or Todd [18] for the third).

TheOREM (Oxtoby). If a topological space is pseudocomplete, then it is a Baire space.

Theorem 1.4. (Oxtoby). Any completely metrizable topological space or locally compact Housdorff space is pseudocomplete.

Theorem 1.5. (Oxtoby). The Cartesian product of pseudocomplete spaces is pseudocomplete.

Other presently known permanence properties of pseudocompleteness are undisturbed, yet the change does ease the establishment of additional interesting properties.

2. Quasiregular spaces and pseudocompleteness. Here we discuss the incidence of the quasiregular property and some properties which when associated with it imply pseudocompleteness.

A regular space is clearly quasiregular. The usefulness of the 
quasiregular property is that it gives just the local property needed for the standard Baire category argument but without the emphasis on points that regularity has. The following gives an example of a space that is quasiregular yet has no point at which there is a neighborhood base consisting of closed sets.

EXAMPLE 2.1. Let $X$ be the set of real numbers and $\sigma$ be the topology on $X$ generated by basic open sets of the form $[a, b) \backslash D$ where $D$ is a countable subset of the half-open interval $[a, b)=$ $\{x \in X: a \leqq x<b\}$ and $D \backslash[a, a+1 / n)$ is finite for all $n=1,2,3, \cdots$. Now suppose, for some $\varepsilon>0,[a, a+\varepsilon) \backslash E$ is contained in $[a, b) \backslash D$ with $D$ infinite and $a \notin D$. But $\operatorname{cl}_{\sigma}([a, a+\varepsilon) \backslash E)=[a, a+\varepsilon)$ and this meets $D$. Thus $\mathrm{cl}_{\sigma}([a, a+\varepsilon) \backslash E)=[a, a+\varepsilon)$ is not contained in $[a, b) \backslash D$, so that $X$ is not regular at $a$. On the other hand, let $a^{\prime}=(\sup D+b) / 2$, then $\left[a^{\prime}, b\right)$ is a nonempty open subset of $X$ that is closed and contained in $[a, b) \backslash D$. Therefore $(X, \sigma)$ is a quasiregular space that is regular at no point.

The next item is part of the folklore.

Proposition 2.2. A dense subspace of a quasiregular space $(Y, \tau)$ quasiregular.

Proof. Let $X$ be a dense subset of $(Y, \tau)$ and let $U$ be in $(\tau \mid X)^{*}$. There is $W$ in $\tau^{*}$ so that $W \cap X=U$. As $(Y, \tau)$ is quasiregular, there is $V$ in $\tau^{*}$ with $\operatorname{cl}_{\tau} V$ contained in $W$, and so $V \cap X$ is in $(\tau \mid X)^{*}$ with $\operatorname{cl}_{\tau \mid X}(V \cap X) \subset\left(\operatorname{cl}_{\tau} V\right) \cap X \subset W \cap X=U$. Therefore $(X, \tau \mid X)$ is quasiregular.

An extension of a quasiregular space need not be quasiregular as the next example shows. By an extension of a topological space $X$, we mean a pair $\langle Y, f\rangle$ where $f$ is a dense embedding of $X$ in the topological space $Y$. A compactification of $X$ is an extension $\langle Y, f\rangle$ of $X$ with $Y$ compact. However, a one-point compactification of $X$ is obtained by appending to $X$ an object $\infty$ that is, not in $X$ and taking as the topology of $Y=X \cup\{\infty\}$ all open subsets of $X$ and all complements in $Y$ of closed compact subsets of $X$ (see Kelley [10] p. 150).

EXAMPLE 2.3. Let $Y=Q \cup\{\infty\}$ be a one-point compactification of the rational numbers $Q$ with the usual topology. Now $Q$ is a nonempty open subset of $Y$, and we suppose that $U$ is a nonempty open subset of $Y$ that is contained in $Q$. $U$ is open in $Q$. If $K$ is a subset of $Q$ that is closed and compact in $Q$, then $K$ is closed and 
compact in the reals $R$. As $Q$ is dense in $R, K$ is rare in $Q$ and so $U \not \subset K$. Therefore $U$ meets $Y \backslash K$, and so $\infty$ is in $\operatorname{cl}_{Y} U$. Thus no nonempty open subset of $Y$ has its closure contained in $Q$. The space $Y$ is not quasiregular, yet it is an extension of a regular (in fact, completely normal) Hausdroff space $Q$.

A topological space $X$ is $H$-closed if any continuous image in a Hausdorff space is closed. It is well-known (Liu [13]) that this is characterized by the following: Each filterbase $\mathscr{U}$ in the lattice of open sets of $X$ has an accummulation point, i.e., $\cap\{\operatorname{cl} U: U$ in $\mathscr{U}\} \neq \phi$. Hajek and Todd [7] have shown that a similar property characteriizes the lightly compact spaces (those spaces for which each locally finite family of open sets is finite (Bagley, Connell, and McKnight [3]): A space $X$ is lightly compact if each countable filterbase in the lattice of open sets has an accummlation point. Also in [7], an infra $H$-closed space was defined to be a space for which any continuous image of it in a first countable Hausdorff space is closed. We have the following implications:

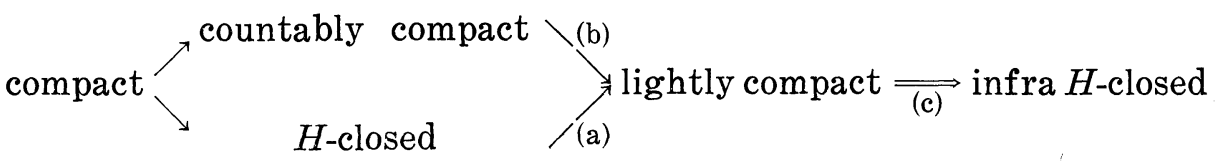

Implications (a) and (c) are discussed in [7]. Bagley, Connell and McKnight [3] cite an example of a lightly compact topological space that is locally compact and completely regular yet not countably compact. This also shows the strictness of (a) as a regular $H$-closed space is compact. Implication (b) is also shown to be strict from Example 2.5 which gives a quasiregular $H$-closed Hausdorff space that has a sequence $((n, 0))_{n}$ with no accummulation point. We now connect this with pseudocompleteness.

Proposition 2.4. If a quasiregular space $X$ is lightly compact, then it is pseudocomplete.

Proof. Let each $\mathscr{B}_{n}$ equal the set of all nonempty open sets of $X$. If $B_{n}$ is in $\mathscr{B}_{n}$ and int $B_{n}$ contains cl $B_{n+1}$, then (int $\left.B_{n}\right)_{n}$ is a countable filterbase in the lattice of open sets of the lightly compact space $X$, so $\bigcap_{n} B_{n}$ contains the nonempty set $\bigcap_{n} \mathrm{cl} B_{n+1}$. Since $X$ is also quasiregular, it is pseudocomplete from Definition 1.5.

Example 2.3 shows that a lightly compact space need not be a pseudocomplete or even a Baire space.

Bagley, Connell and McKnight [3] show the following: A topological space is lightly compact if and only if each proper subset $S$ 
with $S=$ cl int $S$ is lightly compact. Thus, from Proposition 2.4, the closure of any open subset of a lightly compact space is pseudocomplete. Of course this is a property of any pseudocomplete space, in fact, for an open subset $U$ of a pseudocomplete space, if $U \subset X \subset$ cl $U$, then $X$ is a pseudocomplete subspace.

As an $H$-closed space is compact if and only if it is regular, it is interesting to consider an example of Willard ([20] p. 127, Prop. 17K. 4):

EXAMPLE 2.5. Let $N$ be the natural numbers, $N^{*}=\{1 / n: n$ in $N\} \cup\{0\}$ with their usual subspace topologies, and $X=N \times N^{*} \cup\{\infty\}$ where $U$ is an open subset of $X$ if and only if $U$ is an open subset of $N \times N^{*}$ with its product topology or $U \backslash\{\infty\}$ is open in $N \times N^{*}$ and $U$ contains $U_{k}=\{(n, 1 / m): m, n$ in $N$ and $n \geqq k\} \cup\{\infty\}$ for some natural number $k$. Now $X$ is Hausdorff and, in fact, $H$ closed. The latter may be verified by using the following characterization of $H$-closed spaces (see Liu [13]): A space is $H$-closed if and only if each open cover has a finite subcollection whose union is dense. Each cl $U_{k}$ contains the point $(k, 0)$ so that $X$ is not regular at the point $\infty$, and thus is not compact. The set of isolated points of $X$ is $N \times\{1 / m: m$ in $N\}$ which is dense in $X$, so that, as $X$ is Hausdorff, each nonempty open set $U$ contains a clopen set $\{x\}$. Thus $X$ is quasiregular, and since it is lightly compact it is pseudocomplete by Proposition 2.4. Therefore the Hausdorff space $X$ is a noncompact $H$-closed space that is pseudocomplete.

An infra $H$-closed space, even if it is regular, need not be pseudocomplete or even Baire as is now shown.

EXAMPLE 2.6. The space $X$ discussed in Hajek and Todd [7] is a slight modification of an example of Herrlich [8]. This modification gives a regular Hausdorff space with the property that any continuous image of $X$ in any first countable Hausdorff space is a singleton. Therefore $X$ is an infra $H$-closed space. From the original construction $X=\bigcup_{n} X_{n}$ where a subset $U$ of $X$ is defined to be open if and only if $U \cap X_{n}$ is. open in $X_{n}$ for each $n=1,2,3, \cdots$. Moreover, each $X_{n}$ is a closed subset of $X_{n+1}$ with empty interior in $X_{n+1}$. It is now simple to show that each $X_{n}$ is rare in $X$, so that $X=\bigcup_{n} X_{n}$ is meager $\left(\left(X \backslash X_{n}\right)_{n}\right.$ is a sequence of open dense sets with empty intersection). Therefore $X$ is a Hausdorff infra $H$-closed space that is regular and not a Baire space.

That an $H$-closed space may be very close to being pseudocomplete but fail to be so because it is not quasiregular will be seen 
shortly. First here is a simple device for enlarging a topology $\tau$ on a set $X:$ For any subset $S$ of $X$ let $\tau(S)=\{U \cup V \cap S: U, V$ in $\tau$ \}. It is easily shown that $\tau(S)$ is a topology on $X$ that is strictly larger than $\tau$ if $S$ is not in $\tau$. Such enlargements have been studied by Levin [12].

ExAMPLE 2.7. Let $S$ be a dense subset of a topological space $(X, \tau)$ and $\sigma=\tau(S)$. A little work shows that $\mathrm{cl}_{\tau} W=\mathrm{cl}_{\sigma} W$ for any $W$ in $\sigma$. Using the characterization of $H$-closed spaces given in Example 2.5, we see that $(X, \sigma)$ is $H$-closed if $(X, \tau)$ is. Also if $X \backslash S$ is $\tau$-dense, $(X, \sigma)$ is not quasiregular in a rather catastrophic way. For suppose $W_{1}$ is in $\sigma^{*}$. Then $W_{2}=W_{1} \cap S$ is in $\sigma^{*}$. For $W=U \cup V \cap S$ in $\sigma^{*}, \operatorname{cl}_{\sigma} W=\operatorname{cl}_{\tau} W=\operatorname{cl}_{\tau}(U \cup V \cap S) \supset \operatorname{cl}_{\tau}((U \cup V) \cap S)=$ $\operatorname{cl}_{\tau}(U \cup V)$ contains elements of the $\tau$-dense set $X \backslash S$. Therefore $W_{2}$ does not contain $\mathrm{cl}_{\sigma} W$. Thus each $W_{1}$ in $\sigma^{*}$ contains some $W_{2}$ in $\sigma^{*}$ that does not contain the $\sigma$-closure of any nonempty $\sigma$-open set.

In particular, let $X=[0,1], \tau$ be the usual topology and $S$ be the set of irrational numbers in $X$. Both $\tau$ and $\sigma=\tau(S)$ coincide on $S$ which has a complete metric (Kelley [10] p. 207 Prop. 6. $\mathrm{K}(\mathrm{b})$ ) and so is pseudocomplete. $(X, \tau)$ is compact, so $(X, \sigma)$ is $H$-closed. Now $X \backslash S$ is $\tau$-dense, and so $(X, \sigma)$ is not quasiregular, although it is an extension of a pseudocomplete space and so is a Baire space. Therefore $(X, \sigma)$ is a Hausdorff $H$-closed space that is not quasiregular yet is a Baire space since it is an extension of a pseudocomplete space.

3. A relation on the topologies for a set. Our definition of pseudobase allows a simple description of a close relationship between topologies on a set: Topologies $\tau$ and $\sigma$ on a set $X$ are $S$-related if $\tau^{*}$ is a pseudobase for $\sigma$. Later this will aid in extending a theorem of Oxtoby. Here we discuss implications based on this relation.

Proposition 3.1. The S-relation on the topologies for a set $X$ is an equivalence relation.

Proof. From the definition of pseudobase we see that $\tau S \sigma$ means: For all $U$ and $V$ in $\tau^{*}$ and $\sigma^{*}$ respectively, int $\sigma \neq \phi$ and $\operatorname{int}_{\tau} V \neq \phi$. This gives an equivalence relation on the topologies for set $X$.

The usual and the Sorgenfrey topologies on the real line are $S$-related, yet are very different topologies. The topologies in the next example, though homeomorphic, give quite different $S$-related 
topologies.

ExAMPLE 3.2. Let $\sigma$ be the Sorgenfrey topology on the real line, that is, the basic open sets for $\sigma$ are of the form $[a, b)$. Let $\tau$ be the topology on the line with basic open sets of the form ( $a$, $b]$. Each of these topologies are S-related to the usual topology of the line and so are $S$-related to each other. Moreover neither topology is finer than the other, $\sigma \cap \tau$ is the usual topology on the line and $\sigma \vee \tau$, the topology generated by their union, is the discrete topology.

The next proposition emphasizes the closeness of S-related topologies.

Proposition 3.3. If $\sigma$ and $\tau$ are topologies on a set $X$ and $\tau^{*}$ is a pseudobase for $\sigma$, then for any subset $A$ of $X$,

$$
\operatorname{int}_{\sigma} A \subset \mathrm{cl}_{\sigma} \operatorname{int}_{\tau} A \text { and } \operatorname{cl}_{\sigma} A \supset \operatorname{int}_{\sigma} \operatorname{cl}_{\tau} A \text {. }
$$

Proof. Let $x$ be in $\operatorname{int}_{\sigma} A$ and $W$ be a $\sigma$-open neighborhood of $x$. Now $U=\operatorname{int}_{\tau}\left(W \cap \operatorname{int}_{\sigma} A\right) \neq \phi$, and the $\tau$-open set $U$ is contained in $A$, so $W$ meets $\operatorname{int}_{\tau} A$. Thus $x$ is in $\operatorname{cl}_{\sigma} \operatorname{int}_{\tau} A$ and so $\operatorname{int}_{\sigma} A$ is contained in $\mathrm{cl}_{\sigma}$ int $_{\tau} A$. Using $\mathscr{C} A=X \backslash A$, for each subset $A$ of $X$, we have $\mathscr{C} \operatorname{int}_{\sigma} \mathrm{cl}_{\tau} A=\mathscr{C} \mathscr{C} \mathrm{cl}_{\sigma} \mathscr{C} \mathscr{C}$ int $_{\tau} \mathscr{C} A=\mathrm{cl}_{\sigma}$ int $_{\tau} \mathscr{C} A$ which contains int $\sigma \mathscr{C} A$ by the first part of the proposition. Taking complements, we obtain $\operatorname{int}_{\sigma} \mathrm{cl}_{\tau} A \subset \mathscr{C} \operatorname{int}_{\sigma} \mathscr{C} A=\operatorname{cl}_{\sigma} A$, which is the second inclusion of the proposition.

If one member of an $S$-equivalence class is a Baire space then all members are by the following:

Proposition 3.4. Suppose $\sigma$ and $\tau$ are topologies on a set $X$. If $\tau^{*}$ is a pseudobase for $\sigma$ and $(X, \tau)$ is a Baire space, then $(X, \sigma)$ is a Baire space.

Proof. Let $V$ be a dense open set of $(X, \sigma)$ and $U=\operatorname{int}_{\tau} V$. Using both inclusions of Proposition 3.3, we obtain $X=\mathrm{cl}_{\sigma} V=\mathrm{cl}_{\sigma}$ int $_{\sigma} V \subset \mathrm{cl}_{\sigma}$ int $_{\tau} V=\mathrm{cl}_{\sigma} U$, giving $\mathrm{cl}_{\tau} U \supset$ int $_{\tau} \mathrm{cl}_{\sigma} U \supset$ int $_{\tau} X=X$. Therefore $U$ is a dense open set of $(X, \tau)$. Now suppose $\left(V_{n}\right)$ is a sequence of dense open sets of $(X, \sigma)$ and $V$ is in $\sigma^{*}$. Let $U_{n}=$ $\operatorname{int}_{\tau} V_{n}$ and $U=\operatorname{int}_{\tau} V$. Since each $U_{n}$ is a dense open set of the Baire space $(X, \tau)$, and $U$ is a nonempty $\tau$-open set, $U$ meets $\bigcap_{n} U_{n}$. Therefore $V(\supset U)$ meets $\bigcap_{n} V_{n}\left(\supset \bigcap_{n} U_{n}\right)$, and so $\bigcap_{n} V_{n}$ is dense in $(X, \sigma)$. Hence $(X, \sigma)$ is a Baire space. 
From this proposition, we see immediately that the Sorgenfrey line is a Baire space as its topology is $S$-related to the usual topo$\log y$, which is completely metrizable.

The next several propositions use a stronger connection between topologies than that of being $S$-related.

Proposition 3.5. Suppose a topological space $(Y, \tau)$ is the image under a continuous function $f$ of a topological space $(X, \sigma)$ and each member of $\sigma^{*}$ contains a member of $f^{-1} \tau \backslash\{\phi\}$. If $(Y, \tau)$ is quasiregular than $(X, \sigma)$ is quasiregular.

Proof. Let $V$ be in $\sigma^{*}$. There is $U$ in $\tau^{*}$ with $f^{-1} U \subset V$ and $W$ in $\tau^{*}$ with $\operatorname{cl}_{\tau} W \subset U$, so that $\phi \neq f^{-1} W=$ int $_{o} f^{-1} W \subset f^{-1} \mathrm{cl}_{\tau} W \subset$ $f^{-1} U \subset V$. Since $f^{-1} \mathrm{cl}_{\tau} W$ is $\sigma$-closed, $f^{-1} W$ is a nonempty $\sigma$-open set whose $\sigma$-closure is contained in $V$. Therefore $(X, \sigma)$ is quasiregular.

CoRollary 3.6. Suppose $\sigma$ and $\tau$ are topologies on a set $X, \tau$ is coarser than $\sigma$ and each member of $\sigma^{*}$ contains a member of $\tau^{*}$. If $(X, \tau)$ is quasiregular, then $(X, \sigma)$ is quasiregular.

Proof. Apply the previous proposition for $f$ the inclusion map of $(X, \sigma)$ in $(X, \tau)$.

Proposition 3.7. Hypotheses are as in Corollary 3.6. If $(X, \tau)$ is quasiregular and $(X, \sigma)$ is pseudocomplete, then $(X, \tau)$ is pseudocomplete.

Proof. Suppose $\left(\mathscr{B}_{n}\right)$ is a sequence of pseudobases of $\sigma$ verifying the pseudocomplete property for $(X, \sigma)$. Since each member of $\tau^{*}$ is a member of $\sigma^{*}$ and each member of $\sigma^{*}$ contains a member of $\tau^{*}$ the topologies $\sigma$ and $\tau$ are $S$-related. Thus $\sigma^{*}$ is a pseudobase for $\tau$, and it is simple to verify that each $\mathscr{B}_{n}$ is a pseudobase for $(X, \tau)$. Now suppose $B_{n} \in \mathscr{B}_{n}$ with

$$
\text { int }_{\tau} B_{n} \supset \mathrm{cl}_{\tau} B_{n+1} \text {. }
$$

Then as $\tau \subset \sigma$, we have

$$
\operatorname{int}_{\sigma} B_{n} \supset \operatorname{int}_{\tau} B_{n} \text { and } \operatorname{cl}_{\tau} B_{n+1} \supset \mathrm{cl}_{\sigma} B_{n+1},
$$

so that

$$
\operatorname{int}_{\sigma} B_{n} \supset \mathrm{cl}_{\sigma} B_{n+1},
$$

and thus $\bigcap_{n} B_{n} \neq \phi$. Therefore, as $(X, \tau)$ is quasiregular, it is 
pseudocomplete.

A slightly different way of looking at this result is the following.

CoRollaRy 3.8. If a quasiregular topological space $(Y, \tau)$ is the image under a continuous one-to-one function $f$ of a pseudocomplete topological space $(X, \sigma)$ and $f \sigma \backslash\{\phi\}$ is a pseudobase for $\tau$, then $(Y, \tau)$ is pseudocomplete.

Proof. The topology $\eta=f^{-1} \tau$ on $X$ is contained in $\sigma$. Also $(X, \eta)$ is quasiregular. Now for $V$ in $\sigma^{*}, U=\operatorname{int}_{\tau} f V \neq \dot{\phi}$, and, since $f$ is one-to-one, $f^{-1} U$ is in $\eta^{*}$ with $f^{-1} U \subset V$. Thus $\eta \subset \sigma$ and each member of $\sigma^{*}$ contains a member of $\eta^{*}$ so Proposition 3.7 applies, and $(X, \eta)$ is pseudocomplete. Now $(Y, \tau)$ is homeomorphic to $(X, \eta)$, so $(Y, \tau)$ is pseudocomplete.

Note that the requirement that $f \sigma \backslash\{\phi\}$ be a pseudobase is weaker than requiring $f$ to be both continuous and open.

We offer two ways of obtaining topologies $\tau$ and $\sigma$ on a set $X$ with $\tau \subset \sigma$ and $\tau^{*}$ a pseudobase of $\sigma$.

EXAmple 3.9. Let $\tau$ be a topology on $X$ and $S$ a subset of $X$ such that $S \subset \operatorname{cl}_{\tau} \operatorname{int}_{\tau} S$. Let $\sigma=\tau(S)=\{U \cup V \cap S: U, V$ in $\tau\}$. As in Example 2.7, $\tau \subset \sigma$, strictly if $S$ is not in $\tau$. Now for $U \cup V \cap$ $S \neq \phi$ either $\phi \neq U \subset U \cup V \cap S$ or $\phi \neq V \cap$ int $_{\tau} S \subset U \cup V \cap S$, so that $\tau^{*}$ is a pseudobase for $\sigma$.

The next example is related to Example 2.1.

EXAMPLE 3.10. Suppose $\tau$ is a topology on a set $X$ with each $U$ in $\tau^{*}$ uncountable. Let

$$
\mathscr{V}=\left\{U \backslash D: U \in \tau, D \subset U, D \text { countable, } D^{d} \text { finite }\right\},
$$

where $A^{d}$ is the derived set of a subset $A$ of $X$, that is, the set of all points $x$ in $X$ such that $x$ is in $\operatorname{cl}_{\tau}(A \backslash\{x\})$. Now as $\left(U_{1} \backslash D_{1}\right) \cap$ $\left(U_{2} \backslash D_{2}\right)=U_{1} \cap U_{2} \backslash\left(D_{1} \cup D_{2}\right)$, it is easily seen that $\mathscr{V}$ is in fact, a base for a topology $\sigma$ on $X$. Also $U \backslash\left(D \cup D^{d}\right)=U \backslash \mathrm{cl}_{\tau} D \subset U \backslash D$, and clearly $\tau \subset \sigma$, so $\tau^{*}$ is a pseudobase for $\sigma$. (Here it is useful to have each $U \in \tau^{*}$ uncountable, $D$ countable, and $D^{d}$ finite.) By Corollary 3.6, $(X, \sigma)$ is quasiregular if $(X, \tau)$ is quasiregular. Note that if $a$ is a nonisolated point of $(X, \tau)$ at which $\tau$ is first countable, then $(X, \sigma)$ is not regular at $a$. 
4. Lightly compact pseudobases and pseudocompleteness. In this section we shall use the relation discussed in the previous section and light compactness to extend a theorem of Oxtoby [16] on topological spaces with pseudobases of closed countably compact sets.

Theorem 4.1. Suppose $X$ is a dense $G_{j}$-subset of a quasiregular topological space $(Y, \tau)$ that has a pseudobase $\mathscr{C}$ of lightly compact sets. If $\sigma$ is a topology on $Y$ finer than $\tau$ with $\tau^{*}$ as a pseudobase, then the subspace $(X, \sigma \mid X)$ is a pseudocomplete space.

Proof. Let $C$ be in $\mathscr{C}$. As $(Y, \tau)$ is quasiregular, there is $U$ in $\tau^{*}$ with $\operatorname{cl}_{\tau} U \subset \operatorname{int}_{\tau} C$. The set $\operatorname{cl}_{\tau} U$ is lightly compact since $C$ is lightly compact, $\operatorname{cl}_{\tau} U=\left(\mathrm{cl}_{\tau} U\right) \cap C=\mathrm{cl}_{\tau \mid C} U$, and $U \in \tau \mid C$. We may therefore assume that $\mathscr{C}$ consists of closed lightly compact sets of $(Y, \tau)$. Since $\tau \subset \sigma$ and $\tau^{*}$ is a quasibase for $\sigma$, the topological space $(Y, \sigma)$ is quasiregular by Corollary 3.6. Also $\mathrm{cl}_{\sigma} X \supset$ int $_{\sigma} \mathrm{cl}_{\tau} X=$ int $_{\sigma}$ $Y=Y$, so that the dense subspace $(X, \sigma \mid X)$ of $(Y, \sigma)$ is quasiregular by Proposition 2.2.

Now let $\left(G_{n}\right)$ be a sequence of $\tau$-open sets with $X=\bigcap_{n} G_{n}$ and let $\mathscr{B}_{n}=\left\{C \cap X: C \in \mathscr{C}\right.$ and $\left.C \subset G_{n}\right\}$. For $C \cap X$ in $\mathscr{B}_{n}$, we have $\phi \neq \operatorname{int}_{\tau} C \subset \operatorname{int}_{\sigma} C$, so $\phi \neq\left(\operatorname{int}_{\sigma} C\right) \cap X \subset \operatorname{int}_{\sigma \mid X}(C \cap X)$. For $V$ in $\sigma^{*}$, there is $U$ in $\tau^{*}$ with $U \subset V$ and $C$ in $\mathscr{C}$ with $C \subset U \cap G_{n}$ so that $C \cap X \in \mathscr{B}$ and $C \cap X \subset U \cap X \subset V \cap X$. We have shown that each $\mathscr{B}_{n}$ is a pseudobase for $(X, \sigma \mid X)$.

Suppose $B_{n} \in \mathscr{B}_{n}$ and int ${ }_{o \mid X} B_{n} \supset \mathrm{cl}_{\sigma \mid X} B_{n+1}$. There are $C_{k}$ in $\mathscr{C}$ with $C_{k} \subset G_{k}$ and $C_{k} \cap X=B_{k}$. Let $V_{k} \in \sigma$ be such that $V_{k} \cap X=\operatorname{int}_{\sigma \mid X} C_{k} \cap X$. Now $\bigcap_{k=1}^{n} V_{k} \supset \bigcap_{k=1}^{n} V_{k} \cap X=V_{n} \cap X \neq \phi$ and $C_{k}=\operatorname{cl}_{\sigma} C_{k} \supset \operatorname{cl}_{\sigma}\left(V_{k} \cap X\right)=$ $\operatorname{cl}_{\sigma} V_{k} \supset V_{k}$, so that $\bigcap_{k=1}^{n} \operatorname{int}_{\tau} C_{k} \supset \operatorname{int}_{\tau} \bigcap_{k=1}^{n} V_{k} \neq \phi$. Therefore $\left\{\bigcap_{k=1}^{n}\right.$ int $\left._{\tau} C_{k}\right\}_{n \geqq 1}$ is a countable filterbase of open sets of the lightly compact $\tau$-closed subspace $\left(C_{1}, \tau \mid C_{1}\right)$, and so $\bigcap_{n} C_{n} \supset \bigcap_{n}$ cl $_{\tau}$ int $_{\tau} C \neq \dot{\phi}$. But each $C_{n} \subset G_{n}$ so that $\bigcap_{n} C_{n} \subset \bigcap_{n} G_{n}=X$, and $\bigcap_{n} B_{n}=\bigcap_{n} C_{n} \cap X=$ $\left(\bigcap_{n} C_{n}\right) \cap X=\bigcap_{n} C_{n} \neq \phi$. Therefore $(X, \sigma \mid X)$ is a pseudocomplete space.

The following is an immediate application of the theorem and will be used later.

COROLlary 4.2. A topological space with a pseudobase of closed lightly compact sets is pseudocomplete.

As a quasiregular lightly compact space has a pseudobase of closed lightly compact sets Proposition 2.4 is a corollary of the above. 
CoRollary 4.3. Suppose $(Y, \tau)$ has a pseudobase $\mathscr{C}$ of closed lightly compact sets. If $\sigma$ is a topology on $Y$ finer than $\tau$ with $\tau^{*}$ as a pseudobase, then any dense $G_{\delta}$-subspace of $(Y, \sigma)$ is pseudocomplete.

Proof. First note a few facts: By Corollary $4.2(Y, \tau)$ is pseudocomplete, and so, by Theorem 1.3 it is a Baire space. By Proposition $3.4,(Y, \sigma)$ is also a Baire space. From the proof of that proposition, the $\tau$-interior of a dense open subset of $(Y, \sigma)$ is dense in $(Y, \tau)$ and vice versa.

Let $Z$ be a dense $G_{\delta}$-subspace of $(Y, \sigma)$. There is a sequence $\left(H_{n}\right)$ of dense open sets of $(Y, \sigma)$ with $Z=\bigcap_{n} H_{n}$. Using the above noted facts, we see that $X=\bigcap_{n}$ int $_{\tau} H_{n}$ is a dense $G_{\delta}$-subset of $(Y, \tau)$. As it contains $\bigcap_{n}$ int $_{o}$ int $_{\tau} H_{n}$, which is dense in $(Y, \sigma), X$ is also dense in $(Y, \sigma)$.

From Theorem 4.1, $(X, \sigma \mid X)$ is a pseudocomplete space. By Corollary 3.6, $(Y, \sigma)$ is quasiregular, and so, by Proposition 2.2, $(Z$, $\sigma \mid Z)$ is quasiregular. Thus $(Z, \sigma \mid Z)$ is a quasiregular extension of the pseudocomplete space $(X, \sigma \mid X)$ and is, itself, pseudocomplete by Proposition 5.3.

It turns out that the Sorgenfrey line is pseudocomplete in Oxtoby's sense (for each $\mathscr{B}_{n}$ use the family of nonempty basic open sets $[a, b)$ with rational endpoints for even $n$ and irrational for odd $n$ ). We get the following from the previous corollary.

Corollary 4.4. Each dense $G_{\dot{o}}$-subspace of the Sorgenfrey line is pseudocomplete.

Proof. Let $Y$ be the set of real numbers, $\tau$ be the usual topology, $\sigma$ be the Sorgenfrey topology, and $\mathscr{C}$ be the family of $\tau$-compact intervals $[a, b]$ with $a<b$. Now $\mathscr{C}$ is a pseudobase for $\tau$ consisting of $\tau$-lightly compact $\tau$-closed intervals, $\sigma$ is finer than $\tau$ and $\tau^{*}$ is a pseudobase for $\sigma$. Corollary 4.3 applies, and so any dense $G_{i}$-subspace of $(Y, \sigma)$ is pseudocomplete.

The following leads to interesting Sorgenfrey type topologies to which the theorem may be applied.

Example 4.5. Let $X=R \times R$ be the Cartesian plane with $\tau$ as the usual product topology. The family of sets of the form

$$
(a, b) \times(c, d) \cup\{(a, c)\}
$$

is a base for a topology $\sigma$ on $X$ that is stronger than $\tau$ for which 
$\tau^{*}$ is a pseudobase. Moreover, $\mathscr{C}=\{[a, b] \times[c, d]: a, b, c, d \in R, a<$ $b, c<d\}$ is a pseudobase for $\tau$ of $\tau$-closed and $\tau$-compact sets. By Theorem 4.1, $(X, \sigma)$ is pseudocomplete. (The same conclusion may be obtained for topologies based on $\sigma$ as in Example 3.10). It may be noted that $(X, \sigma)$ is strictly stronger than the product topology on $X$ when each factor is given the Sorgenfrey topology. This product topology is pseudocomplete by Corollary 4.4 and Theorem 1.5 .

In another direction we shall improve on the Baire category theorem of Hajek [6]. An extension $\langle Y, f\rangle$ of a topological space $X$ is a relatively Hausdorff extension if $f x$ and $y$ have disjoint neighborhoods whenever $x$ is in $X, y$ is in $Y$ and $f x \neq y$.

Proposition 4.6. If $Y$ is a relatively Hausdorff extension of a topological space $X$ and each point of a dense subset $S$ of $Y$ has a neighborhood whose closure is compact, then $Y$ has a pseudobase of closed compact sets. Moreover $Y$ is pseudocomplete.

Proof. For convenience, we consider $X$ as a dense subspace of $(Y, \tau)$, so that the relatively Hausdroff property means that elements, $x$ and $y$ of $X$ and $Y$ respectively, have disjoint neighborhoods whenever $x \neq y$. Let $\mathscr{B}=\left\{\right.$ cl $U: U \in \tau^{*}$ and cl $U$ is compact $\}$. We shall show that $\mathscr{B}$ is a pseudobase for $\tau$. Let $W$ be in $\tau^{*}$. As $S$ is dense in $(Y, \tau)$ there is an $s$ in $W \cap S$. There is an open neighborhood $U$ of $s$ with cl $U$ compact. As $X$ is dense in $Y$, there is an $x$ in $U \cap W \cap X$. Let $F=(\operatorname{cl} U) \backslash W$.

Since $Y$ is a relatively Hausdorff extension of $X$, for each $y$ in $F$, there are disjoint open neighborhoods $U_{y}$ and $V_{y}$ of $x$ and $y$ respectively. As the open sets $\left\{V_{y}: y \in F\right\}$ cover the compact set $F$, there is a finite subcollection $\left\{V_{y^{(k)}}\right\}_{k=1}^{n}$ that covers $F$. Let $U_{0}=$ $\bigcap_{k=1}^{n} U_{y(k)} \cap U \cap W$, so that $U_{0}$ is an open neighborhood of $x$ that is contained in $U$ and disjoint from the open neighborhood $V=\bigcap_{k=1}^{n}$ $V_{y(k)}$ of $F=(\operatorname{cl} U) \backslash W$. Thus $U_{0} \subset(\operatorname{cl} U) \backslash V \subset W$. Since $(\operatorname{cl} U) \backslash V$ is closed, $W$ contains cl $U_{0}$. Moreover, as cl $U$ is compact, so is cl $U_{0}$. Finally $x$ is in $U_{0}$, so $U_{0}$ is in $\tau^{*}$, and therefore cl $U_{0}$ is a member of $\mathscr{B}$ that is contained in $W$. Also each member of $\mathscr{B}$ has nonempty interior, so $\mathscr{B}$ is a pseudobase for $\tau$ consisting of closed compact sets, and from Corollary $4.2(Y, \tau)$ is pseudocomplete.

Hajek [5] shows that a topological space is regular and Hausdorff if and only if it has a relatively Hausdorff compactification. Then in [6], Hajek shows that such an extension of a nonempty topological space is nonmeager. This is now strengthened in the 
following immediate result.

Corollary 4.7. A relatively Hausdorff compactification $\langle Y, f\rangle$ of a topological space $X$ has a pseudobase of closed compact sets. Moreover $Y$ is pseudocomplete.

5. Extensions. In this section are discussed some results on extensions and pseudocompleteness including a characterization of spaces with quasiregular one-point compactifications.

After constructing, for each topological space $X$, a Baire extension $X_{F}$, McCoy [14] asks if each regular space has a regular Baire extension. In this context, a natural modification of this question is to replace "regular" by "quasiregular". It is easy to see that McCoy's Baire extension $X_{F}$ of $X$ is quasiregular if $X$ is, so the modified question has an affirmative answer. Aarts and Lutzer [1] give a stronger result by constructing, for each quasiregular space, a pseudocomplete extension. This extension is, in fact, an $H$-closed extension, and so we may characterize quasiregular spaces in a manner similar to Hajek's [5] characterization of regular Hausdorff spaces: A topological space is quasiregular if and only if it has a quasiregular $H$-closed extension. In case, the space is $T_{1}$ (singletons are closed), we have the following.

Proposition 5.1. A $T_{1}$-space $(X, \tau)$ is quasiregular if and only if it has a quasiregular compactification.

Proof. One implication is clear as a dense subspace of a quasiregular space is quasiregular. We shall be using the Wallman compactification $\left\langle W(X), \varphi_{X}\right\rangle$ of the $T_{1}$-space $(X, \tau)$ so we note: (i) $W(X)=$ $\{\mathscr{F}$ an ultrafilter in the lattice of closed sets of $(X, \tau)\}$, (ii) a basis for the closed sets of $W(X)$ is the family of all sets of the form $C(A)=\{\mathscr{F} \in W(x): A \in \mathscr{F}\}$ where $A$ is closed in $(X, \tau)$, and (iii) the dense embedding $\varphi_{X}$ of $X$ in $W(X)$ is given by $\varphi_{X}(x)=\{A \subset X$ : $X \backslash A \in \tau$ and $x \in A\}$ (see Kelley [10] p. 167, prob. 6.R.) Now let $V$ be a nonempty open subset of $W(X)$, so there is a proper closed set $A$ of $X$ with $W(X) \backslash C(A) \subset V$. As $(X, \tau)$ is quasiregular, there is $U$ in $\tau^{*}$ with $\operatorname{cl}_{\tau} U \subset X \backslash A$. We need only show that

$$
W(X) \backslash C(X \backslash U) \subset C\left(\operatorname{cl}_{\tau} U\right) \subset W(X) \backslash C(A) .
$$

Suppose $\mathscr{F}$ is in $W(X) \backslash C(X \backslash U)$. Then $X \backslash U$ is not in $\mathscr{F}$. But, as $(X \backslash U) \cup \operatorname{cl}_{\tau} U=X$, we have $\operatorname{cl}_{\tau} U$ in $\mathscr{F}$ so that $\mathscr{F}$ is in $C\left(\operatorname{cl}_{\tau} U\right)$. Now, as $\operatorname{cl}_{\tau} U \subset X \backslash A, \mathrm{cl}_{\tau} U$ is disjoint from $A$. Therefore $\operatorname{cl}_{\tau} U$ is in no closed ultrafilter containing $A$, and so $\mathscr{F}$ in $C\left(\mathrm{cl}_{\tau} U\right)$, does not contain $A$. Thus $\mathscr{F}$ is not in $C(A)$, and the second inclusion holds. 
Hence $\left\langle W(X), \varphi_{X}\right\rangle$ is a quasiregular compactification of $X$.

Here we shall verify that a quasiregular extension of a pseudocomplete space is pseudocomplete. The argument seems somewhat more delicate that than for which pseudobases are collections of open sets ([1] Prop. 2.2(a)).

LEMMA 5.2. If a quasiregular topological space $(Y, \sigma)$ has a dense subspace $X$ with pseudobase $\mathscr{B}$, then $\mathscr{C}=\{C \subset Y: C \cap X \in \mathscr{B}$, and $\left.\operatorname{int}_{\sigma} C \supset \operatorname{int}_{\sigma \mid X}(C \cap X)\right\}$ is a pseudobase for $(Y, \sigma)$.

Proof. In the above definition of $\mathscr{C}$, each $C \cap X$ is in $\mathscr{B}$ so int $_{\sigma \mid X}(C \cap X)$ is nonempty, and as $\operatorname{int}_{\sigma} C$ contains $\operatorname{int}_{\sigma \mid X}(C \cap X)$, each member of $\mathscr{C}$ has a nonempty $\sigma$-interior. We need to show that each $V$ in $\sigma^{*}$ contains a member of $\mathscr{C}$. As $(Y, \sigma)$ is quasiregular there is $V_{1}$ in $\sigma^{*}$ with $\operatorname{cl}_{\sigma} V_{1} \subset V$. There is $B$ in $\mathscr{B}$ with $B \subset V_{1} \cap X$. Let $W$ be in $\sigma^{*}$ with $W \cap X=\operatorname{int}_{\sigma \mid X} B$, and set $C=W \cup B$. Now $C \cap X=W \cap X \cup B \cap X=\left(\right.$ int $\left._{\sigma \mid X} B\right) \cup B=B \in \mathscr{B}$, and int $_{\sigma} C \supset W \supset W \cap$ $X=\operatorname{int}_{\sigma \mid X} B$, so that $C$ is a member of $\mathscr{C}$. Moreover, $W \subset \mathrm{cl}_{\sigma} W=$ $\left(\operatorname{cl}_{\sigma}(W \cap X)\right) \subset \operatorname{cl}_{\sigma} B \subset \operatorname{cl}_{\sigma}\left(V_{1} \cap X\right)=\operatorname{cl}_{\sigma} V_{1} \subset V$, so that $C=W \cup B \subset V$. Therefore $\mathscr{C}$ is a pseudobase for $Y$.

Now we have the extension property of pseudocompleteness.

Proposition 5.3. If a topological space $\langle Y, f\rangle$ is a quasiregular extension of a pseudocomplete space $X$, then $Y$ is pseudocomplete.

Proof. For convenience, assume that $(X, \tau)$ is a dense pseudocomplete subspace of quasiregular space $(Y, \sigma)$ so that $\tau=\sigma \mid X$. Suppose $\left(\mathscr{B}_{n}\right)$ is a sequence of pseudobases for $(X, \tau)$ verifying that $(X, \tau)$ is a pseudocomplete space. Define the sequence $\left(\mathscr{C}_{n}\right)$ by $\mathscr{C}_{n}=$ $\left\{C \subset Y: C \cap X \subset \mathscr{B}_{n}\right.$ and $\left.\operatorname{int}_{\sigma} C \supset \operatorname{int}_{\tau}(C \cap X)\right\}$. By Lemma 5.2 each $\mathscr{C}_{n}$ is a pseudobase for $(Y, \sigma)$. Suppose $C_{n} \in \mathscr{C}_{n}$ with $\operatorname{int}_{\sigma} C_{n} \supset \mathrm{cl}_{\sigma} C_{n+1}$, and note that $B_{n}=C_{n} \cap X \in \mathscr{B}_{n}$. Now $B_{n}=C_{n} \cap X \supset\left(\right.$ int $\left._{\sigma} C_{n}\right) \cap X \supset$ (int $\left.B_{n}\right) \cap X=\operatorname{int}_{\tau} B_{n}$, so that int $B_{n}=\left(\right.$ int $\left._{\sigma} C_{n}\right) \cap X \supset\left(\operatorname{cl}_{\sigma} C_{n+1}\right) \cap X \supset$ $\left(\operatorname{cl}_{\sigma} B_{n+1}\right) \cap X=\operatorname{cl}_{\sigma} B_{n+1}$. Therefore, by assumption, $\bigcap_{n} B_{n} \neq \dot{\phi}$, and so $\bigcap_{n} C_{n} \neq \phi$. Thus $(Y, \sigma)$ is a pseudocomplete space.

Topological spaces in which compact sets are closed are called KC-spaces. The following definition will be useful to us: An extension $\langle Y, f\rangle$ of a topological space $X$ is called a relatively $\mathrm{KC}$ extension if $f K$ is closed in $Y$ for each compact subset $K$ of $X$. 
Proposition 5.4. If a topological space $(X, \tau)$ has a pseudobase $\mathscr{B}$ of compact sets and $\langle Y, f\rangle$ is a relatively KC-extension of $X$ then there is a dense open subset $U$ of $Y$ contained in the embedding $f X$ of $X$ in $Y$. Moreover, $f \mathscr{B}$ is a pseudobase for $Y$ of closed compact subsets.

Proof. We shall treat $(X, \tau)$ as a dense subspace of $(Y, \sigma)$ so that $\tau=\sigma \mid X$. Let $U=\cup\left\{\right.$ int $\left._{\sigma} B: B \in \mathscr{B}\right\}$. Clearly $U$ is $\sigma$-open and contained in $X$. Let $V$ be in $\sigma^{*}$. Since $X$ is $\sigma$-dense, there is a member $B$ of $\mathscr{B}$ contained in $V \cap X$ and $\operatorname{int}_{\sigma} \operatorname{cl}_{\sigma} B$ is nonempty. Since $B$ is compact and $Y$ is a relatively KC-extension of $X, \operatorname{cl}_{\sigma} B=B$. Now $\phi \neq \operatorname{int}_{\sigma} \operatorname{cl}_{\sigma} B=$ int $_{\sigma} B \subset B \subset V \cap X$, so $V$ meets $U$. Therefore $U$ is a $\sigma$-dense open set of $Y$ that is contained in $X$. It is clear that $\mathscr{B}$ is a pseudobase for $(Y, \sigma)$ consisting of $\sigma$-closed $\sigma$-compact sets.

The next gives a simple kind of converse to the above and will not be proved.

Proposition 5.5. If a topological space $(Y, \sigma)$ has a pseudobase $\mathscr{C}$ of closed (lightly) compact sets, then for any open set $U$ and any subset $X$ of $Y$ with $U \subset X \subset \mathrm{cl}_{\sigma} U$, the subspace $(X, \sigma \mid X)$ has a pseudobase of closed (lightly) compact sets.

We now consider some results connected with one-point compactifications.

Proposition 5.6. If a topological space $(X, \tau)$ has a pseudobase $\mathscr{B}$ of closed compact sets, then the one-point compactification $X^{*}=$ $X \cup\{\infty\}$ of $X$ has $\mathscr{B}$ as a pseudobase of closed compact sets.

Proof. If $(X, \tau)$ is compact, the result is simple. Suppose otherwise. Since each member $B$ or $\mathscr{B}$ is closed and compact in $(X, \tau)$, $X^{*} \mid B$ is, by definition, open in $X^{*}$. Therefore $B$ is a closed subset of $X^{*}$. It was at this point in the proof of Proposition 5.4 that the relatively KC-property was used, so this present proposition is essentially a corollary of that proposition.

By a locally compact space is meant a space such that each point has a compact neighborhood.

Proposition 5.7. If a topological space $(X, \tau)$ is quasiregular and locally compact, then it has a pseudobase of closed compact sets. 
Proof. Let $\mathscr{B}=\left\{\mathrm{cl} V: V \in \tau^{*}\right.$ and $\mathrm{cl} V$ is compact $\}$. Suppose $U$ is in $\tau^{*}$ and $x$ is in $U$. There is a compact neighborhood $N$ of $x$. Since $N \cap U$ is a neighborhood of $x$, there is a $V$ in $\tau^{*}$ such that cl $V$ is contained in (int $N) \cap U$. Thus the closed set cl $V$ is compact and is a member of $\mathscr{B}$ that is contained in $U$. Therefore $\mathscr{B}$ is a pseudobase for $(X, \tau)$ of closed compact sets.

We may now give a characterization of topological spaces with one-point compactifications that are quasiregular.

THeOREM 5.8. The one-point compactification $X^{*}=X \cup\{\infty\}$ of a topological space $(X, \tau)$ is quasiregular if and only if $(X, \tau)$ has a pseudobase of closed compact sets.

Proof. Suppose that $X^{*}$ is quasiregular. Since it is compact, it has a pseudobase $\mathscr{C}$ of closed compact sets by Proposition 5.7. Now $X$ is an open subspace of $X^{*}$ so that $\mathscr{B}=\{C \subset X: C \in \mathscr{C}\}$ is a pseudobase for $(X, \tau)$ of closed compact sets of $(X, \tau)$. Conversely, suppose $(X, \tau)$ has a pseudobase $\mathscr{B}$ of closed compact sets. By Proposition $5.6, X^{*}$ has a pseudobase of closed compact sets, and so is quasiregular.

6. Questions. The notion of pseudocompleteness discussed here forms an answer to the unification problem mentioned in $\S 1$. A related question is what permanence properties does such a unifying property share with the Baire property. Aarts and Lutzer $[1,2]$ cite two specific questions for Oxtoby's notion of pseudocompleteness that also remain open for the notion defined in this paper:

Question 6.1. Is a dense $G_{i}$-subspace of a pseudocomplete space pseudocomplete?

Question 6.2. Is the open continuous image of a pseudocomplete space pseudocomplete?

Some partial results for these are given in $\S \S 3$ and 4 , and, in a much restricted form, the second question has also been considered in . Todd [18].

The following remains open:

Question 6.3. Is the notion of pseudocompleteness discussed here equivalent to Oxtoby's pseudocompleteness?

Oxtoby [16] discusses countability conditions on pseudobases. 
Pseudobases consisting of sets satisfying a compactness condition have been discussed here. What other interesting local properties might be usefully expressed in terms of pseudobases?

For more on Baire extensions, see R. A. McCoy and J. Porter, General Topology and its Applications, 7 (1977), 34-58.

\section{REFERENCES}

1. J. M. Aarts and D. J. Lutzer, Pseudocompleteness and the product of Baire spaces, Pacific J. Math., 48 (1973), 1-10.

2. — Completeness properties designed for recognizing Baire spaces, Dissert. Math., 96 (1974).

3. R. W. Bagley, E. H. Connell and J. D. Macknight, Jr., On properties characterizing pseudo-compact spaces, Proc. Amer. Math. Soc., 9 (1958), 500-506.

4. W. G. Fleissner and K. Kunen, Barely Baire spaces, Fund. Math., 101 (1978), 229-240.

5. D. W. Hajek, A characterization of $T_{3}$-spaces, Indiana U. J. Math., 23 (1973), $23-25$.

6. - Relatively Hausdorff compactifications are second category, Nanta Math., 10 (1977), 67.

7. D. W. Hajek and A. R. Todd, Lightly compact spaces and infra H-closed spaces, Proc. Amer. Math. Soc., 48 (1975), 479-482.

8. H. Herrlich, Wann sind alle stetigen Abbildungen in Y Konstant? Math. Zeit., 90 (1965), 152-154.

9. H. Herrlich, Nich alle $T_{2}$-minimalen Raume sind von 2. Kategorie, 91 (1966), 185. 10. J. L. Kelley, General Topology, D. Van Nostrand, Princeton, New Jersey, 1955.

11. J. L. Kelley, Namioka, et al., Linear Topological Spaces, D. Van Nostrand, Princeton, New Jersey, 1963.

12. N. Levine, Simple extensions of topologies, Amer. Math. Monthly, 71 (1964), 22-25. 13. Chen-Tung Liu, Absolutely closed spaces, Trans. Amer. Math. Soc., 130 (1968), 86-104.

14. R. A. McCoy, A Baire space extension, Proc. Amer. Math. Soc., 33 (1972), 199-202. 15. - A filter characterization of regular Baire spaces, Proc. Amer. Math. Soc., 40 (1973), 268-270.

16. J. C. Oxtoby, Cartesian products of Baire spaces, Fund. Math., 49 (1961), 157-166. 17. R. Pol, Note on category in Cartesian products of metrizable spaces, Fund. Math., 102 (1979), 55-59.

18. A. R. Todd, Continuous linear images of pseudo-complete linear topological spaces, Pacific J. Math., 63 (1976), 281-292.

19. —_ Pseudocompleteness in linear metrizable spaces, Fund. Math., 94 (1977), 93-96.

20. S. Willard, General Topology, Addison-Wesley, Reading, Mass., 1970.

Received October 12, 1979

ST. Johns UnIVERSITY

Staten Island, NY 10301 


\section{PACIFIC JOURNAL OF MATHEMATICS}

\section{EDITORS}

DONALD BABBITT (Managing Editor)

University of Galifornia

Los Angeles, California 90024

Hugo RossI

University of Utah

Salt Lake City, UT 84112

C. C. MOORE AND ANDREW OGG

University of California

Berkeley, CA 94720
J. DugundJI

Department of Mathematics University of Southern California Los Angeles, California 90007

R. FinN and J. Milgram Stanford University Stanford, California 94305

\section{ASSOCIATE EDITORS}

R. ARENS

E. F. BeCKenbaCh

B. H. NEUManN

F. WOLF

K. YosHIDA

\section{SUPPORTING INSTITUTIONS}

UNIVERSITY OF ARIZONA

UNIVERSITY OF BRITISH COLUMBIA

CALIFORNIA INSTITUTE OF TECHNOLOGY

UNIVERSITY OF CALIFORNIA

MONTANA STATE UNIVERSITY

UNIVERSITY OF NEVADA, RENO

NEW MEXICO STATE UNIVERSITY

OREGON STATE UNIVERSITY
UNIVERSITY OF OREGON

UNIVERSITY OF SOUTHERN CALIFONIA

STANFORD UNIVERSITY

UNIVERSITY OF HAWAII

UNIVERSITY OF TOKYO

UNIVERSITY OF UTAH

WASHINGTON STATE UNIVERSITY

UNIVERSITY OF WASHINGTON 


\section{Pacific Journal of Mathematics}

\section{Vol. 95, No. $1 \quad$ September, 1981}

John Allen Beachy and William David Blair, On rings with bounded

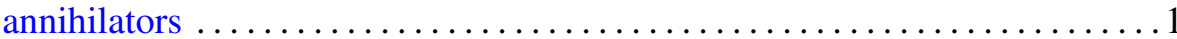

Douglas S. Bridges, A constructive look at positive linear functionals on

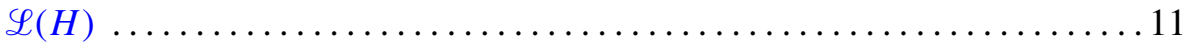

Muneo Chō and Makoto Takaguchi, Boundary points of joint numerical ranges

W. J. Cramer and William O. Ray, Solvability of nonlinear operator equations

Lester Eli Dubins and Gideon Schwarz, Equidiscontinuity of

Borsuk-Ulam functions

Maria Fragoulopoulou, Spaces of representations and enveloping 1.m.c.

*-algebras

Robert F. Geitz and J. Jerry Uhl, Jr., Vector-valued functions as families of scalar-valued functions

Ross Geoghegan, The homomorphism on fundamental group induced by a homotopy idempotent having essential fixed points

Ross Geoghegan, Splitting homotopy idempotents which have essential fixed points

Paul Jacob Koosis, Entire functions of exponential type as multipliers for

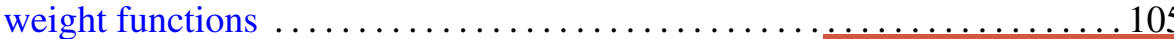

David London, Monotonicity of permanents of certain doubly stochastic matrices

Howard J. Marcum, Two results on cofibers

Giancarlo Mauceri, Zonal multipliers on the Heisenberg group

Edward Wilfred Odell, Jr. and Y. Sternfeld, A fixed point theorem in $c_{0} \quad \ldots 161$

Bernt Karsten Oksendal, Brownian motion and sets of harmonic measure zero

Andrew Douglas Pollington, The Hausdorff dimension of a set of normal numbers

Joe Repka, Base change lifting and Galois invariance ...

Gerald Suchan, Concerning the minimum of permanents on doubly stochastic circulants

Jun-ichi Tanaka, On isometries of Hardy spaces on compact abelian groups

Aaron R. Todd, Quasiregular, pseudocomplete, and Baire spaces 